\title{
Proposta de um algorítmo para seleção de coberturas, segundo o tipo de lesão aberta em crianças*
}

\author{
Proposal of an algorithm for dressings selection, according to skin lesions in children \\ La propuesta de un algoritmo para la selecion de curar, segundo el tipo de lesiones superficiales en \\ los niños
}

\author{
Gabrilelle Tayar ${ }^{1}$, Maria Angelica Sorgini Peterlini' ${ }^{2}$, Mavilde da Luz Gonçalves \\ Pedreira $^{2}$
}

\section{RESUMO}

Objetivos: Este estudo descritivo teve por objetivos elaborar um algoritmo para apoiar a decisão da enfermeira na seleção de coberturas segundo o tipo de lesão aberta em crianças e avaliar sua aplicabilidade, segundo a opinião de enfermeiras pediátricas. Métodos: O algoritmo desenvolvido é composto por determinantes relativos à avaliação do tecido e estágio de cicatrização, limpeza da ferida e tipo de cobertura primária e secundária utilizado. Resultados: Entre as 24 enfermeiras que participaram da avaliação do algoritmo, o tempo de formadas e de trabalho em pediatria apresentou mediana de dois anos; 87,5\% eram pós-graduandas e 100\% cuidavam de uma a quatro crianças com feridas abertas por mês. Quanto a avaliação do algoritmo, na maioria das variáveis verificadas, o instrumento foi considerado ótimo. Conclusão: Após a utilização do algoritmo proposto, 95,8\% das enfermeiras consideraram um importante instrumento para aplicação na prática assistencial.

Descritores: Enfermagem; Enfermagem pediátrica; Bandagens; Cicatrização de feridas

\begin{abstract}
Objectives: This descriptive study aimed to elaborate an algorithm to support the nurses' selection of dressings for treatment in different wounds in children and to evaluate its effectiveness according to pediatric nurses' opinion. Methods: This algorithm was composed of steps related to assessment of skin and healing process stage, the cleaning process determination and primary and secondary dressings selection. Results: The 24 nurses who participated in the algorithm evaluation were graduated at two years in average and since then they have been working in the pediatric field; $87,5 \%$ had post graduation courses and all of them have been caring by month, a rate of one to four children with open wounds. Regarding to the algorithm evaluation, in the majority of studied variables, the nurses described the protocol as useful to support nursing interventions. Conclusion: The majority $(95,8 \%)$ of the nurses described the instrument as important to application in practice.
\end{abstract}

Keywords: Nursing; Pediatric nursing; Bandages; Wound healing

\section{RESUMEN}

Objetivos: Este estudio descriptivo tenido por objetivos elaborar un algoritmo para apoyar la decisión de la enfermera en la selecion del cuidado en los tipos diferentes de heridas presentado por los niños y evaluar la pertinencia practica, a través de la aplicación de un instrumento de la investigación. Métodos: El algoritmo desarrollado y compuesto para el determinante relativa la limpieza de la herida, la evaluación clinica de aprendiz de cicatrisacion y tipo de primero y secundario curativo el ellos se usen. Resultados: Los 24 enfermeras que participaron en la evaluación del algoritmo ellas poseyeron el mediana de dos años de formación y trabajan en la pediatría; $87,5 \%$ eran postgraduadas y $100,0 \%$ tuvieron el cuidado en el período de trabajo, de uno a cuatro niños con las heridas abiertas un mes. Como la evaluación del algoritmo, en la mayoría de las variables verificadas, el instrumento fue considerado grande. Conclusíon: 95,8\% de las enfermeras consideraron el instrumento importante para la aplicación en la práctica.

Descriptores: Enfermería; Enfermería pediátrica, Vendajes; Cicatrización de heridas

\footnotetext{
*Trabalho realizado na Escola Paulista de Enfermagem - Universidade Federal de São Paulo - UNIFESP. Monografia de conclusão do Curso de Especializaçãa em Enfermagem - Modalidade Residência - Área de Concentração Pediatria Geral

${ }^{1}$ Pós-graduanda do Programa de Pós-Graduação em Enfermagem da Universidade Federal de São Paulo - UNIFESP, São Paulo (SP), Brasil.

${ }^{2}$ Doutora, Professora Adjunto da Disciplina Enfermagem Pediátrica da Universidade Federal de São Paulo - UNIFESP, São Paulo (SP), Brasil.
} 


\section{INTRODUÇÃO}

O cuidado de ferida faz parte das atribuições da enfermeira, sendo que a abrangência desta atuação vem crescendo progressivamente nos últimos anos, devido ao maior conhecimento referente ao processo de cicatrização dos tecidos e o desenvolvimento científico e tecnológico do cuidado de enfermagem prestado a pacientes que apresentam lesões de pele.

Em neonatos e crianças uma ferida aberta pode ser causada por injúrias traumáticas como: pressão do probe do oxímetro, inadequada fixação da cânula orotraqueal, sondas e cateteres, remoção incorreta de adesivos e fixações, além de acidentes da infância e automobilísticos; injúrias químicas por extravasamento de soluções hipertônicas ou vesicantes e as infecciosas como celulites, entre outras, propiciando a ação de bactérias como Pseudomonas aeruginosa ${ }^{(1)}$. A presença de uma ferida aberta em criança é causa de dor, privação de atividade, e quando não tratada adequadamente pode prolongar o período de hospitalização, propiciando a exposição à infecções e procedimentos invasivos, muitas vezes traumáticos.

Durante a pós-graduação em Enfermagem Pediátrica, o confronto com o cuidado de enfermagem a crianças portadoras de feridas levou ao desejo de compreender a temática e as possibilidades terapêuticas que aceleram a cicatrização na criança, para reduzir o tempo de hospitalização, minimizar o risco de infecção, dor e traumas.

Para cuidar de criança portadora de ferida é necessário compreender a estrutura do sistema tegumentar, o processo de cicatrização e a definição dos tipos de feridas.

A pele é o maior órgão do corpo, fornece proteção, atua como barreira química e mecânica, é órgão sensorial vital para a percepção de pressão, dor e temperatura, participando da termorregulação, excreta água e eletrólitos e sintetiza vitamina $\mathrm{D}^{(2-4)}$. É composta por duas camadas que se encontram firmemente aderidas uma a outra, a epiderme e a derme, que se sobrepõem ao tecido subcutâneo ${ }^{(3,5)}$.

Qualquer lesão no tecido epitelial que acarreta prejuízo para o desempenho de suas funções chama-se ferida ${ }^{(6-8)}$.

A ferida pode ser definida como o resultado de ilimitada variedade de injúrias traumáticas, isquêmicas, cirúrgicas ou pressóricas que agridem a estrutura histofisiológica do tecido tegumentar ${ }^{(9)}$.

A classificação das feridas, embora bastante variável, é uma importante forma de sistematização para o processo de avaliação e cuidado. Sendo assim, o tempo de reparação tissular pode ser um dos tipos de classificação, e consiste em agudas e crônicas. As feridas agudas são resultantes de cirurgias e traumas (mais comum em crianças), cuja reparação ocorre em seqüência e tempo adequado, sem complicação, levando à restauração da integridade anatômica e funcional. As crônicas, por sua vez, são aquelas que não são reparadas em tempo adequado e apresentam complicações ${ }^{(10)}$.

Segundo o Pressure Ulcer Scale for Healing ${ }^{(11-12)}$, as feridas crônicas também podem ser classificadas de acordo com a aparência do leito da lesão, definida como o tipo de tecido prevalente nessa região, sendo especificada como: tecido necrótico, esfacelo, tecido de granulação e tecido de epitelização ${ }^{(13)}$.

A presença de uma ferida estimula imediatamente uma série de processos metabólicos, visando o restabelecimento dos tecidos lesados ${ }^{(14-15)}$.

O processo de cicatrização da ferida pode ser subdividido em fases altamente complexas, interdependentes e sobrepostas: fase inflamatória, proliferativa ou reconstrutiva e a de maturação. Estas fases possuem durações variadas, sendo que qualquer uma pode prolongar-se devido a fatores locais ou sistêmicos que acarretam comprometimentos ${ }^{(10,16)}$.

O processo de cicatrização pode ocorrer por primeira e segunda intenção ${ }^{(8-9)}$. A cicatrização por primeira intenção ocorre quando há perda de tecido e as bordas são próximas ou passíveis de ajuste por sutura. $\mathrm{Na}$ cicatrização por segunda intenção, ocorre perda acentuada do tecido e não há possibilidade de fechamento das bordas necessitando da manutenção do meio úmido ${ }^{(8-9,17-18)}$. A função microcirculatória desempenha importante papel na reparação tecidual, propiciando a chegada de células de defesa na área afetada, com conseqüente eliminação do agente agressor, por permitir a migração de células fagocitárias e enzimas proteolíticas, promovendo o desbridamento e a limpeza da lesão, além de prover condições necessárias para a formação de tecidos ${ }^{(17)}$.

Como demonstrou Ovington ${ }^{(19)}$, a infecção é um fator muito comum que atrasa o fechamento de feridas crônicas e agudas, pois está associada com os níveis de bactérias viáveis nos tecidos, uma vez que secretam exotoxinas e podem produzir endotoxinas, resultante de sua lise. Essas toxinas tendem a causar necrose local e a interferir no balanço de mediadores (citocinas e proteases) necessários para a progressão da cicatrização.

Para propiciar e acelerar o processo de cicatrização utilizam-se coberturas, que se caracterizam como meios terapêuticos aplicados a uma ferida previamente limpa, cujo objetivo principal é a eliminação de fatores negativos que possam retardar a cicatrização( ${ }^{(7)}$.

Os curativos podem ser subdivididos em primários, aqueles colocados diretamente sobre a ferida e na pele adjacente, e secundários quando instalados sobre o curativo primário.

Para uma cicatrização adequada é necessário conhecer as coberturas disponíveis no mercado, tal como sua composição, mecanismo de ação, indicação, advertências, 
modo de usar e o tempo de troca.

A enfermeira tem importante papel no tratamento da criança portadora de ferida aberta, pois é o profissional que avalia, prescreve e realiza este cuidado. Atualmente, existe uma diversidade de dispositivos para curativos no mercado, que devem adequar-se às características de cada tipo de lesão aberta. Por vezes, observa-se a implementação de diferentes tipos de coberturas para curativos em um mesmo tipo de lesão, em um mesmo serviço, sendo que, nem sempre, o cuidado aplicado supre a necessidade da criança.

Por este motivo, surgiu o desejo de "uniformizarmos" este cuidado, por meio da elaboração de um instrumento para direcionar e apoiar a decisão da enfermeira na determinação da cobertura e cuidado adequado a crianças portadoras de lesão aberta.

\section{OBJETIVOS}

Os objetivos deste estudo foram:

- Elaborar um algorítmo para apoiar a decisão da enfermeira na seleção da cobertura para curativo, segundo diferentes tipos de lesões de pele em crianças.

- Verificar a aplicabilidade prática do algorítmo elaborado, segundo a opinião de enfermeiras pediatras.

\section{MÉTODOS}

Trata-se de um estudo descritivo, com levantamento de estudos das bases de dados Lilacs, Medline, Cochrane, Cinahal, além de consultas bibliográficas a livros e teses da área dos últimos 10 anos, utilizando como palavraschave: pediatria, curativos, pele, ferida e cuidados de enfermagem.

O estudo foi submetido à apreciação do Comitê de Ética em Pesquisa das Instituições envolvidas recebendo parecer favorável de $\mathrm{n}^{\circ}$ 0719/02.

Os locais do estudo foram dois hospitais da cidade de São Paulo, sendo um universitário.

A partir deste levantamento elaborou-se um algorítmo (Figura 1), para apoiar a decisão da enfermeira na seleção de coberturas segundo diferentes tipos de lesões de pele em crianças, com intuito de uniformizar a assistência prestada. Utilizaram-se fotos de feridas de crianças que estiveram previamente sob cuidado das pesquisadoras, após a assinatura, pelos responsáveis, do Termo de Consentimento Livre e Esclarecido, para facilitar a visualização e identificação da evolução das lesões, seguindo a seqüência da cicatrização: tecido necrótico, esfacelo, de granulação e de epitelização ${ }^{(11-13)}$.

Assim que o algorítmo foi elaborado, realizou-se a avaliação da sua aplicabilidade prática por meio de um questionário, composto por variáveis referentes a identificação da enfermeira e a verificação da aplicabilidade prática do algorítmo quanto a apresentação gráfica, facilidade de leitura, seqüência lógica, pertinência da descrição dos tipos de tecidos e curativos e apoio a decisão da enfermeira durante a escolha do curativo, cujas categorias representadas foram de ótimo, bom, regular e ruim, além de campos abertos para sugestões.

A população do estudo foi composta por 27 enfermeiras, sendo estas residentes, docentes de enfermagem pediátrica e a enfermeira responsável pela assistência em cada unidade pediátrica das instituições investigadas. Entretanto, como três enfermeiras não concordaram em participar da coleta de dados, a amostra ficou constituída por 24 profissionais.

\section{RESULTADOS}

Por meio da literatura pesquisada e dos estudos de casos realizados pelos profissionais das instituições participantes da pesquisa encontramos a fundamentação necessária para a elaboração do algorítmo.

No algorítmo proposto, a enfermeira ao avaliar a necessidade de implementar o cuidado com uma ferida aberta na criança, independente do tipo de lesão, realiza a limpeza da mesma com soro fisiológico (SF) morno e em jato de $12 \mathrm{psi}$ (seringa de $20 \mathrm{ml}$ com agulha 40x12), para propiciar o desbridamento mecânico eficaz, diminuir o risco de infecção da ferida, prevenir queda de temperatura no leito da ferida e estimular a vasodilatação local ${ }^{(20)}$. Em seguida, avalia a lesão para caracterizar a fase da cicatrização, e os tipos de tecido presentes na lesão: necrótico, esfacelo, granulação ou de epitelização para direcionar sua ação na escolha da cobertura adequada à necessidade da criança ${ }^{(2,16,18)}$.

Posteriormente, avalia a presença ou ausência de exsudato, que direciona a escolha do tipo de cobertura, pois algumas atuam melhor na presença de exsudato e outras não.

As seguintes coberturas para curativos foram escolhidas: hidrocolóide, alginato de cálcio e ácidos graxos essenciais, fundamentados em publicações que descrevem suas composições, indicações e mecanismo de ação. Outro fator a ser considerado relaciona-se à disponibilidade destas nas instituições do estudo.

As coberturas devem ser substituídas de acordo com a evolução da cicatrização e melhora do tecido presente no leito da ferida, sendo preconizada a realização de curativos até o fechamento da lesão. A evolução da cicatrização da ferida determina a seqüência de cuidados e o emprego dos materiais de curativos, que se relacionam com a evolução da lesão e indicação clínica de uso das coberturas para curativos.

Ao final da aplicação do curativo primário mais adequado foi proposta a realização de curativo 
secundário com gaze estéril, coberto por membrana transparente semi-permeável ou fita hipoalergênica.

Abaixo, pode-se visualizar o algorítmo para determinação dos tipos de coberturas para curativos utilizadas em crianças, segundo os tipos de lesões de pele.

Após a elaboração do algorítmo, este foi apresentado às enfermeiras participantes do estudo, junto a um questionário para avaliar sua aplicabilidade prática.

A primeira variável analisada no questionário referese à caracterização da população do estudo, segundo o tempo de formada, tempo de trabalho em pediatria e realização de pós graduação.

Observa-se, na Tabela 1, que a maioria das enfermeiras tem menos de cinco anos de formada. Resultado similar obteve-se quanto ao tempo de trabalho em pediatria. As médias do tempo de formada e de trabalho na área foram iguais a quatro anos, a mediana de dois anos e o desvio padrão de aproximadamente cinco anos.

Apesar da amostra constituir-se, na sua maioria, por profissionais com menos de cinco anos de formação, 87,5\% possuíam cursos de pós-graduação, principalmente lato sensu.

Outra variável analisada foi o número de crianças portadoras de lesões abertas cuidadas pelas enfermeiras durante um mês de trabalho. Doze enfermeiras (50,0\%) referiram realizar em média cuidados a uma criança portadora de lesão aberta por mês, sete enfermeiras $(29,16 \%)$ a duas crianças, duas enfermeiras (8,33\%) a três crianças e outras três $(12,51 \%)$ a quatro crianças portadoras de lesão de pele.

Também foram avaliados as características e os conteúdos do algorítmo elaborado, como demonstra a Tabela 2 .

De acordo com a Tabela 2, a maioria das enfermeiras avaliou o algoritmo como ótimo de acordo com as variáveis apresentadas. As variáveis facilidade de leitura e seqüência do algoritmo foram as que apresentaram melhor nível de avaliação com 79,16\% e 75,0\%, respectivamente. Já, a variável de descrição dos tipos de tecidos obteve 8,33\% de avaliação regular, sendo a variável com maior freqüência da categoria regular. Destaca-se que nenhuma das variáveis medidas foi categorizada como ruim, segundo a amostra estudada.

$\mathrm{Na}$ seqüência do instrumento de coleta de dados, perguntou-se às enfermeiras se consideravam o algorítmo importante para a prátic e, 23 profissionais $(95,84 \%)$ responderam que sim.

Obtivemos como sugestões para alteração do algoritmo, a mudança de cores nos quadros que compõem a figura.

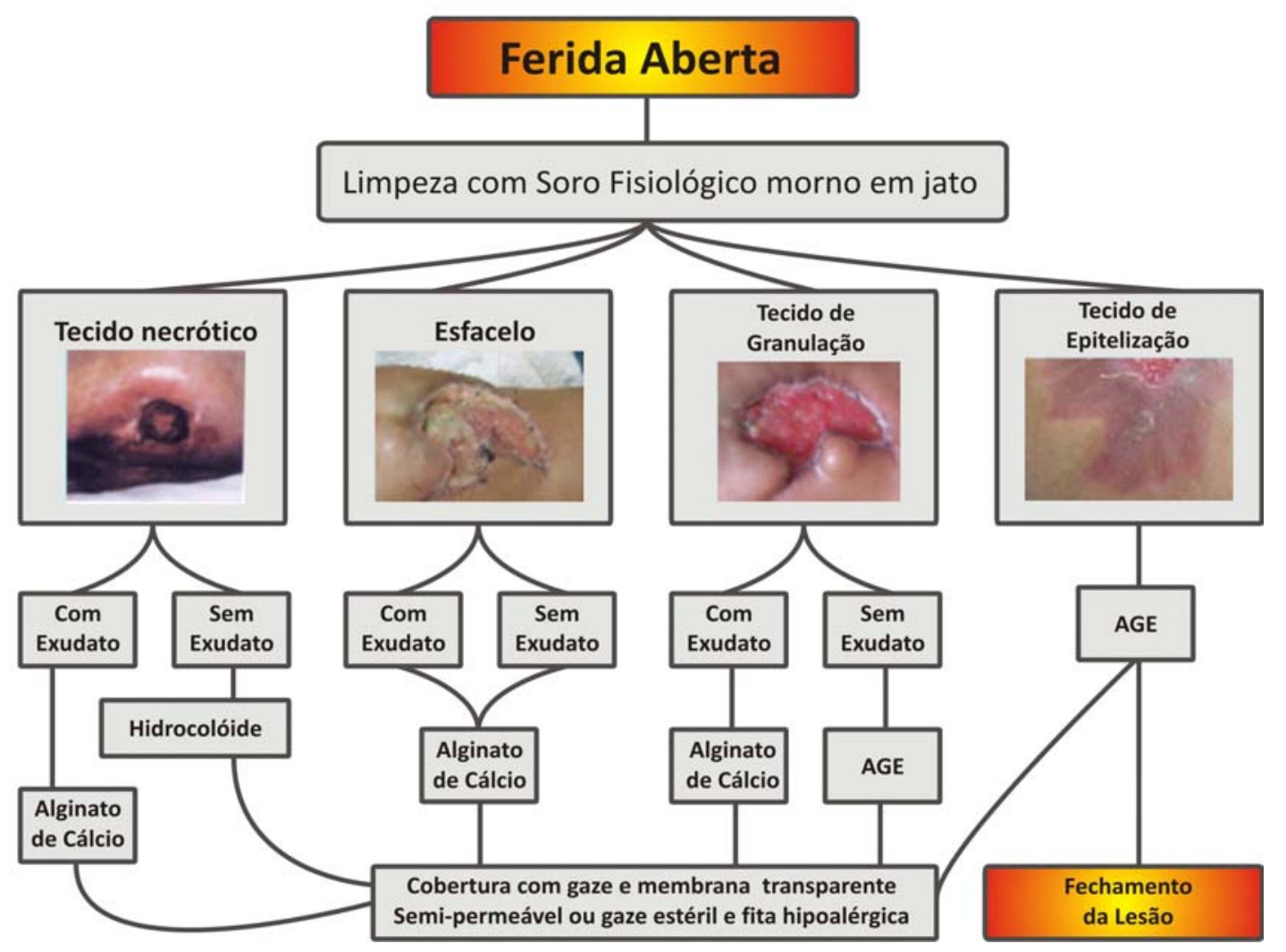

Figura 1 - Algorítimo - Feridas 
Tabela 1 - Caracterização das enfermeiras.

\begin{tabular}{|c|c|c|}
\hline Caracterização das enfermeiras & $\mathbf{N}$ & $\%$ \\
\hline \multicolumn{3}{|l|}{ Tempo de Formada } \\
\hline$<1$ ano & 3 & 12,50 \\
\hline $1 \vdash 5$ anos & 16 & 66,67 \\
\hline $5 \mapsto 10$ anos & 3 & 12,50 \\
\hline$>10$ anos & 2 & 8,33 \\
\hline Média & \multicolumn{2}{|c|}{4 anos } \\
\hline Mediana & \multicolumn{2}{|c|}{2 anos } \\
\hline Desvio Padrão & \multicolumn{2}{|c|}{5 anos } \\
\hline \multicolumn{3}{|l|}{ Tempo de Trabalho em Pediatria } \\
\hline$<1$ ano & 3 & 12,50 \\
\hline $1 \vdash 5$ anos & 17 & 70,84 \\
\hline $5 \vdash 10$ anos & 2 & 8,33 \\
\hline$>10$ anos & 2 & 8,33 \\
\hline Média & \multicolumn{2}{|c|}{4 anos } \\
\hline Mediana & \multicolumn{2}{|c|}{2 anos } \\
\hline Desvio Padrão & \multicolumn{2}{|c|}{5 anos } \\
\hline Realização de pós-graduação & & \\
\hline $\operatorname{Sim}$ & 21 & 87,50 \\
\hline Não & 3 & 12,50 \\
\hline \multicolumn{3}{|l|}{ Maior Titulação } \\
\hline Especialista & 17 & 80,96 \\
\hline Mestre & 2 & 9,52 \\
\hline Doutor & 2 & 9,52 \\
\hline
\end{tabular}

\section{DISCUSSÃO}

Optou-se por elaborar um algorítmo, pois estes são mapas gráficos, usados para maior visualização dos componentes e processos de um problema. Algorítmos clínicos possibilitam passos corretos da evolução de um assunto específico no cuidado do paciente ${ }^{(21)}$.

Segundo Beitz, algorítmos têm sido desenvolvidos para cuidar, guiar decisões clínicas e tratamento de feridas agudas e crônicas. São estudos validados e resultados de recomendações baseadas em pesquisas para a prática. $\mathrm{O}$ cuidado da ferida deve conter a limpeza, a hidratação, o cuidado com exsudato e a utilização de coberturas que retém a umidade ${ }^{(22)}$.

Para a limpeza de qualquer tipo de ferida aberta foi proposto no algorítmo o uso de soro fisiológico morno e em jato, para propiciar o desbridamento mecânico da lesão, prevenir a diminuição da temperatura do leito da ferida e estimular a vasodilatação local, acelerando o processo cicatricial ${ }^{(16,18,20,23)}$. A força hidráulica adequada é de 8 psi para a limpeza e pode ser obtida com uma seringa de $35 \mathrm{ml}$ e agulha de calibre 19 (padrão norteamericano). No Brasil não dispomos deste material, portanto os serviços utilizam seringa de $20 \mathrm{ml}$ com agulha 40x12, para realizar o jato ${ }^{(20,23-24)}$.

Hidrocolóides, alginato de cálcio e ácidos graxos essênciais, foram coberturas escolhidas para implementação dos curativos, com base na literatura estudada e na prática institucional, visando acelerar o tempo de cicatrização na criança, reduzir o tempo de hospitalização, minimizar o risco de infecção, dor e traumas.

Coberturas à base de hidrocolóides são compostas por uma camada externa de espuma de poliuretano e uma camada interna tripla constituída de gelatina, que produz a hidrólise parcial do colágeno, agindo como hemostático e absorvente. A pectina, substância mucilaginosa com alto poder de absorção de água, forma um gel com propriedades protetoras, e a carboximetilcelulose sódica proporciona viscosidade e estabilidade à emulsão ${ }^{(1,7-9)}$.

O hidrocolóide realiza desbridamento autolítico do esfacelo, facilitando o desenvolvimento do tecido de granulação mais vascularizado, tem função de impermeabilizar a ferida, proporcionando um meio hipóxico que estimula o desenvolvimento de tecido de granulação e neovascularização, além de manter a umidade local ${ }^{(1,25-26)}$. É indicado para prevenção e tratamento de feridas abertas não infectadas ${ }^{(11,24,26)}$. Deve ser aplicado sobre a ferida previamente limpa, sendo importante pressionar firmemente as bordas e massagear a placa do hidrocolóide, para melhor aderência. Este curativo pode ser trocado a cada 72 horas ou quando necessário ${ }^{(7-9)}$.

Os curativos de alginato de cálcio são compostos por fibras derivadas de algas marinhas marrons (Laminaria hiperborea), constituídas de ácido gulurônico e manurônico, com íons de cálcio ( $80 \%)$ e sódio $(20 \%)$

Tabela 2 - Caracterização e conteúdo do algoritmo, segundo a avaliação das enfermeiras.

\begin{tabular}{|c|c|c|c|c|c|c|}
\hline \multirow{3}{*}{$\begin{array}{c}\text { Caracterização e } \\
\text { Conteúdo do Algoritmo }\end{array}$} & \multicolumn{6}{|c|}{$\begin{array}{c}\text { Avaliação das Características e } \\
\text { Conteúdo do Algoritmo }\end{array}$} \\
\hline & \multicolumn{2}{|c|}{ Ótimo } & \multicolumn{2}{|c|}{ Bom } & \multicolumn{2}{|c|}{ Regular } \\
\hline & $\mathbf{N}$ & $\%$ & $\mathbf{N}$ & $\%$ & $\mathbf{N}$ & $\%$ \\
\hline Apresentação Gráfica & 14 & 58,34 & 9 & 37,50 & 1 & 4,16 \\
\hline Facilidade de Leitura & 19 & 79,16 & 4 & 16,68 & 1 & 4,16 \\
\hline Sequência do Algoritmo & 18 & 75,00 & 5 & 20,84 & 1 & 4,16 \\
\hline Descrição do Tipo de Tecido & 14 & 58,34 & 8 & 33,33 & 2 & 8,33 \\
\hline Descrição do Tipo de Cobertura & 13 & 54,16 & 10 & 41,16 & 1 & 4,16 \\
\hline
\end{tabular}


impregnados em suas fibras ${ }^{(27)}$.

As fibras do alginato de cálcio são capazes de absorver o exsudato das feridas e convertê-lo em um gel, fornece um ambiente úmido e quente na ferida, adequado para a cicatrização ${ }^{(24)}$. Sua ação ocorre pela interação entre o sódio presente no exsudato e no sangue, com o cálcio impregnado no curativo. Esta troca iônica auxilia no desbridamento autolítico, com alta capacidade de absorção, induzindo a hemostasia ${ }^{(7-9,28-29)}$. São indicados para o tratamento de feridas abertas, sangrantes, altamente exsudativas, com ou sem infecção, até a redução do exsudato $^{(9,20)}$.

O alginato de cálcio deve ser colocado sobre a ferida previamente limpa, abrir e remodelar suas fibras no interior da ferida, ocluir com curativo secundário estéril. Pode ser trocado a cada 24 a 72 horas, ou sempre que necessário, não devendo ser utilizados em implantes cirúrgicos, queimaduras de terceiro grau ou em feridas pouco exsudativas $^{(7-9)}$.

Os ácidos graxos essênciais (AGE) são compostos por óleo vegetal à base de ácido linoléico, ácido caprílico, ácido cáprico, vitamina $\mathrm{A}, \mathrm{E}$, além de lecitina de soja, sendo o ácido linoléico importante para o transporte e metabolismo de gorduras, assim como, na manutenção da função e integridade das membranas ${ }^{(24)}$.

Estudos demonstraram que a deficiência do ácido linoléico em crianças, causa déficit de crescimento e diminuição da resistência às infecções ${ }^{(9)}$. A vitamina A favorece a integridade da pele e sua cicatrização. A vitamina $E$ tem função anti-oxidante e protege a membrana celular do ataque de radicais livres. A lecitina de soja protege, hidrata e auxilia na restauração da pele. ${ }^{(9)}$

Os AGE têm a ação de promover a quimiotaxia para os leucócitos, facilitando a entrada de fatores de crescimento na célula, a mitose e a proliferação celular. São indicados para todos os tipos de lesões infectadas ou não, desde que desbridadas previamente. Devem ser instalados sobre a ferida previamente limpa e ocluído com curativo secundário estéril. As trocas deste curativo devem ser realizadas a cada 24 horas ou quando necessário. Os AGE não são agentes desbridantes, porém estimulam o desbridamento autolítico ${ }^{(8-9)}$.

Ao analisarmos a população do estudo, verificamos que praticamente a totalidade possui pós-graduação e prestam cuidados às crianças portadoras de lesões abertas. Estas enfermeiras $(95,84 \%)$ avaliaram o algoritmo como importante ferramenta para auxiliar na tomada de decisão na seleção da cobertura mais adequada, segundo os diferentes tipos de lesões.

Vale ressaltar que nenhuma participante avaliou o algoritmo como ruim, e a maioria classificou a apresentação gráfica, facilidade de leitura, seqüência, descrição do tipo de tecido e cobertura como ótimas.

Portanto esta ferramenta pode ser utilizada na rotina diária da enfermeira que presta cuidado à criança.

\section{CONCLUSÃO}

O algorítmo elaborado foi submetido à avaliação das enfermeiras que participaram do estudo. A maioria da amostra considerou importante a aplicação deste instrumento para apoiar a decisão da enfermeira na seleção do curativo adequado em crianças portadoras de lesões abertas.

\section{REFERÊNCIAS}

1. Irving V, Young T, Atkinson J. Know how: wound management in neonates. Nurs Times. 1996;92(46):74-5.

2. Gogia PP. Fisiologia da cicatrização de feridas. In: Gogia PP. Feridas: tratamento e cicatrização. Rio de Janeiro: Revinter; 2003. p.1-6.

3. Arnold HL, Odom RB, James WD. Doenças da pele de Andrews - Dermatologia clínica. 8 a ed. São Paulo: Manole; 1994. p. 4-14.

4. Lax IP, Alvarez SG, Grun D. Feridas e seus cuidados. São Paulo: Departamento de Serviços Educacionais; 1992.

5. Gartner LP, Hiatt JL. Tratado de histologia em cores. 2a ed. Rio de Janeiro: Guanabara Koogan; 2003. p. 265-79.

6. Mandelbaum SH, Di Santis EP, Mandelbaum MHS. Cicatrização: conceitos atuais e recursos auxiliares - parte I. Anais Bras Dermatol. 2003;78(4):393-408.

7. Declair V. Aplicação do triglicerídeos de cadeia média (TCM) na prevenção de úlceras de decúbito. Rev Bras Enfermagem. 1994;47(1):27-30.

8. Cesaretti IUR. Processo fisiológico de cicatrização da ferida. Pelle Sana. 1998;2:10-2.

9. Bajay HL. Universidade Estadual de Campinas. Hospital das Clínicas. Grupo de Estudos de Feridas. Tratamento de feridas. Campinas: UNICAMP; 1999.

10. Santos VLCG. Alguns aspectos do tratamento de feridas no domicílio. In: Manual de Enfermagem São Paulo. São Paulo: Instituto para o Desenvolvimento da Saúde; 2001. p. 128-36.

11. Thomas DR, Rodeheaver GT, Bartolucci AA, Franz RA, Sussman C, Ferrell BA, et al. Pressure ulcer scale for healing: derivation and validation of the PUSH tool. The PUSH Task Force Adv Wound Care. 1997;10(5):96-101.

12. Maklebust J. PUSH Tool reality check: audience response. Pressure Ulcer Scale for Healing. Adv Wound Care. 1997;10(5):102-6.

13. Santos VLCG, Azevedo MAJ, Silva TS, Carvalho VMJ, Carvalho VF. Adaptação transcultural do pressure ulcer scale for healing (PUSH) para a língua portuguesa. Rev Latinoam Enfermagem. 2005;13(3):305-13.

14. Hess CT, Kirsner RS. Orchestrating wound healing: assessing and preparing the wound bed. Adv Skin Wound Care. 2003; 16(5):246-57; quiz 258-9 .

15. Machado AF, Schneider VB, Peterlini MAS, Silva MLG, Pereira SR. Uso de alginato de cálcio e triglicérides de cadeia média em duas crianças com lesões ulcerativas. Rev Esc Enfermagem USP. 1999;33(N Espec):230.

16. Dealey C. Cuidando de feridas: um guia para as enfermeiras. 2a ed. São Paulo: Atheneu; 2001. 
17. Jorge SA, Dantas SRPE. Abordagem multiprofissional do tratamento de feridas. São Paulo: Atheneu; 2003

18. Carville K. Wound care manual. rev. ed. Australia: Silver Chain Foundation; 1995.

19. Ovington LG. The well-dressed wound: an overview of dressing types. Wounds. 1998;10(Suppl A):1A-11A.

20. Rodeheaver GT. Wound cleansing, wound irrigation, wound disinfection. In: Krasner D, Kane D. Chronic Wound Care: a clinical source book for healthcare professionals. 2nd.ed. Wayne, PA: Health Management Publications; 1997. p. $97-$ 108.

21. Beitz JM, Bates-Jensen B. Algorithms, critical pathways, and computer software for wound care: contemporary status and future potential. Ostomy Wound Manage. 2001;47(4):33-40; quiz 41-2.

22. Beitz JM, van Rijswijk L. Using wound care algorithms: a content validation study. J Wound Ostomy Continence Nurs. 1999;26(5):238-9, 241-9.

23. Yamada BFA. Terapia tópica de feridas: limpeza e desbridamento. Rev Esc Enfermagem USP. 1999;33:133-
40.

24. McGuckin M, Goldman R, Bolton L, Salcido R. The clinical relevance of microbiology in acute and chronic wounds. Adv Skin Wound Care. 2003; 16(1):12-23; quiz 24-5.

25. Campbell KE, Keast D, Woodbury G, Houghton P. Wear time in two hydrocolloid dressings using a novel in-vitro model. Wounds. 2003; 15(2):40-8.

26. Lyndon MJ. Dissolução de coágulos nas feridas e desenvolvimento do tecido de granulação com Duoderm ${ }^{\mathrm{MR}}$. In: Wounds: Wounds: a compendium of clinical research and practice 1989;2( pt I).

27. Limova M. Evaluation of two calcium alginate dressings in the management of venous ulcers. Ostomy Wound Manage. 2003;49(9):26-33.

28. Luccas GC. Tratamento de úlcera isquêmica de extremidade com curativo hidroativo. Cir Vasc Angiol. 1994;10:26-8.

29. Bodero Petit R, D’Pascuale J. Uso de alginato de cálcio en heridas exudativas e infectadas con exposición de fascia, músculo, tendón y hueso. Rev Mex Ortop Traumatol. 1999;13(5):462-9.

\section{ANEXO \\ Questionário para a avaliação do algorítmo de curativo}

I-Identificação da Enfermeira:

1-Tempo de formado:

Tempo de trabalho em pediatria:

Realizou curso de pós-graduação: $\operatorname{sim}($ ) não ( ) Caso sim, qual ?

( )especialização(área)______________ ( )mestrado(área)

( )doutorado(área)_____________________esidência(área)

( )outros(área)

4-Em média, quantos curativos de feridas abertas você realiza em um mês de trabalho?

\section{II- Avaliação do algoritmo de curativos:}

5- Quanto à apresentação gráfica: ótimo ( ) bom ( ) regular ( ) ruim ( )

Sugestões/ Comentários:

6-Quanto a facilidade de leitura: ótimo ( ) bom ( ) regular ( ) ruim ( )

Sugestões/ Comentários:

7- Quanto à seqüência do algorítmo: ótimo ( ) bom ( ) regular ( ) ruim ( )

Sugestões/ Comentários:

8- Quanto à descrição dos tipos de tecidos: ótimo ( ) bom ( ) regular ( ) ruim ( )

Sugestões/ Comentários:

9- Quanto à descrição dos tipos de curativos: ótimo ( ) bom ( ) regular ( ) ruim ( )

Sugestões/ Comentários:

10-Na sua opinião o algoritmo contém informações capazes de apoiar sua decisão durante a escolha do curativo: sim ( $)$ não ( ) Caso não, porque?

11-Caso ache necessário, faça comentários. Obrigada! 